\title{
Toward the Definition of Efficacy and Safety Criteria for Advancing Gene Drive-Modified Mosquitoes to Field Testing
}

\author{
Stephanie L. James, ${ }^{1}$ John M. Marshall, ${ }^{2}$ George K. Christophides, ${ }^{3}$ Fredros O. Okumu, ${ }^{4}$ and Tony Nolan ${ }^{5}$
}

\begin{abstract}
Mosquitoes containing gene drive systems are being developed as complementary tools to prevent transmission of malaria and other mosquito-borne diseases. As with any new tool, decision makers and other stakeholders will need to balance risks (safety) and benefits (efficacy) when considering the rationale for testing and deploying gene drive-modified mosquito products. Developers will benefit from standards for judging whether an investigational gene drive product meets acceptability criteria for advancing to field trials. Such standards may be formalized as preferred product characteristics and target product profiles, which describe the desired attributes of the product category and of a particular product, respectively. This report summarizes discussions from two scientific workshops aimed at identifying efficacy and safety characteristics that must be minimally met for an investigational gene drive-modified mosquito product to be deemed viable to move from contained testing to field release and the data that will be needed to support an application for first field release.
\end{abstract}

Keywords: mosquito, malaria, gene drive, efficacy, biosafety

\section{Introduction}

$\mathbf{M}$ OSQUitoes MODIFIED WITH gene drive systems are being developed as a potential new tool for preventing transmission of mosquito-borne diseases, either by reducing the numbers of targeted mosquito vectors (population suppression) or reducing their ability to transmit the pathogen to humans (population replacement/modification). Gene drive systems provide for preferential (super-Mendelian) inheritance of the introduced genetic trait(s) within interbreeding mosquito populations. These systems can be self-limiting, where effects are intended to be spatially and/or temporally restricted, or self-sustaining, where the introduced trait(s) is intended to establish and spread within the local population and the effect is intended to persist in the environment (World Health Organization [WHO] 2014a).

Theoretical characteristics of self-sustaining gene drive systems have raised hopes for durable protection against disease transmission at low cost (WHO 2014a, Eckhoff et al. 2017,
North et al. 2019). However, these same predictions also have raised concerns among some about the possibility of harms to health or the environment that might be irreversible (National Academies of Science Engineering and Medicine 2016).

This report summarizes discussions from two 2019 workshops that convened knowledgeable scientists from a range of relevant fields to consider the desired characteristics of gene drive-modified mosquitoes that would help developers identify their specific objectives for product efficacy and safety, and decision-makers to determine whether an investigational product is ready to advance to field testing. For simplicity, discussions focused on the case study of selfsustaining (threshold independent or low threshold ${ }^{1}$ ) gene drive systems for control of malaria transmission by

\footnotetext{
${ }^{1}$ Threshold-independent and low threshold drives include those that are expected to initiate spread of the modification in the local population of target mosquitoes from a rare introduction or low initial release frequency (James, et al. 2018)
}

\footnotetext{
${ }^{1}$ Foundation for the National Institutes of Health, North Bethesda, Maryland.

${ }^{2}$ University of California Berkeley, Berkeley, California.

${ }^{3}$ Imperial College London, London, United Kingdom.

${ }^{4}$ Ifakara Health Institute, Ifakara, Tanzania.

${ }^{5}$ Liverpool School of Tropical Medicine, Liverpool, United Kingdom.
}

(C) Stephanie L. James et al. 2020; Published by Mary Ann Liebert, Inc. This Open Access article is distributed under the terms of the Creative Commons Attribution Noncommercial License (http://creativecommons.org/licenses/by-nc/4.0/) which permits any noncommercial use, distribution, and reproduction in any medium, provided the original author(s) and the source are cited. 
Anopheles gambiae mosquitoes in Africa (James et al. 2018), and are extendable to all strains and sibling vector species in the A. gambiae complex where reasonable levels of hybridization or introgression can occur in the wild.

These systems are based on use of driving endonuclease genes (Gantz et al. 2015, Hammond et al. 2016, Godfray et al. 2017, Kyrou et al. 2018) and are predicted to spread from very low initial release frequency. However, it is expected that these conclusions also will be informative for other types of drive systems, such as toxin-antidote-based systems, and other mosquito-borne diseases transmitted by a variety of species, including infections caused by arboviruses.

\section{Discussion}

The first workshop ${ }^{2}$ addressed the entomological and epidemiological efficacy expectations on which to base a decision to advance an investigational gene drive product to first field release, which approximately equate to the criteria that must be minimally met for an investigational product to be deemed viable for further development (often termed the minimally acceptable product for other types of public health tools). The second workshop ${ }^{3}$ considered recommendations for collection of laboratory and field data that could be required to support an application for first field testing of a new investigational gene drive product, including key predictors of entomological and epidemiological efficacy as well as safety for human and animal health and the environment. For these purposes, the product was defined as the mosquito carrying the gene drive construct.

These discussions are expected to contribute to development of preferred product characteristics (PPCs) and target product profiles (TPPs) for gene drive-modified mosquito products.

\section{Epidemiological outcome}

Health benefits. There was substantial discussion over whether the primary efficacy criterion should be based on an epidemiological or entomological outcome. An epidemiological goal is standard in TPPs for other malaria control products, including those intended to reduce parasite transmission as is the case for gene drive-modified mosquitoes (Vontas et al. 2014, WHO 2014b, Burrows et al. 2017, 2018).

Most participants agreed that, while success of initial smallscale field trials of investigational gene drive products should primarily be assessed on the basis of entomological measures (WHO 2014a, James et al. 2018), health benefit is the ultimate goal, and therefore, potential for disease impact is a primary consideration throughout the product development pathway. This is in keeping with the principle of beneficence espoused in the Belmont Report (US Department of Health Education and Welfare 1979), and several participants predicted that the expectation for a positive epidemiological outcome will be required by oversight agencies when gene drive products are to be field tested in areas of human habitation (WHO 2017a).

\footnotetext{
${ }^{2}$ For more information, see https://fnih.org/sites/default/files/final/ pdf/TPP\%20efficacy\%20criteria\%20FINAL.pdf and https://fnih.org/ sites/default/files/final/pdf/Participant\%20list\%20FINAL.pdf

${ }^{3}$ For more information, see https://fnih.org/sites/default/files/final/ pdf/Data\%20and\%20Design\%20Workshop\%20May\%202019\%20 Agenda.pdf and https://fnih.org/sites/default/files/final/pdf/Data\% 20and\%20Design\%20Workshop\%20May\%202019\%20Participant\% 20List.pdf
}

For decision-making on whether to advance an investigational gene drive product to first release, the potential for health benefit would be informed by modeling based on parameters obtained from the laboratory and baseline field data from the proposed release site.

Regarding the minimum potential epidemiological impact that should qualify an investigational product to advance to first release, the Vector Control Advisory Group of the World Health Organization has stated that an intervention could be recommended for operational deployment if it resulted in at least a $30 \%$ reduction in epidemiological outcomes (WHO 2017b). However, it was noted that for some other malaria control interventions, a predicted efficacy of $20 \%$ additional reduction in clinical incidence of malaria, in the presence of other control methods considered standard of care, has been considered a reasonable threshold for continued development (Foy et al. 2019).

Some participants proposed that because of the possible longterm persistence of gene drive systems in the environment, gene drive products should strive for a greater benefit. They suggested aiming for a predicted epidemiological efficacy of $\sim 50 \%$ reduction in clinical incidence of malaria as the threshold for moving a gene drive candidate from the laboratory to field testing. This reduction is approximately equivalent to the observed efficacy of long-lasting insecticidal nets (Yang et al. 2018). Two caveats were noted in support of a less stringent requirement: (1) that the first gene drive investigational product would likely target only one of several malaria vector species and (2) that the relationship between malaria incidence and entomological inoculation rate (EIR) ${ }^{4}$ is nonlinear, such that disease decline can be slow in medium-to-high transmission settings (Smith et al. 2007). Both of these issues should be considered in the choice of sites for initial field testing.

It also must be noted that ongoing efforts to scale up other malaria control methods are expected to create a more demanding environment for demonstrating epidemiological efficacy for new products in the future, since larger trials may be required to confirm a significant reduction in malaria incidence. Given that investigational gene drive products are not expected to be ready for field testing for several years, participants therefore stressed that thresholds for the first openfield trial of an investigational product should not be set so high as to constitute a barrier to continued development if the value of collecting the data and the potential for success are judged to outweigh costs and risks associated with the trial.

It was further noted that a product with epidemiologic efficacy lower than suggested above might still be appealing as a public health tool if its cost is low compared to other tools for malaria control and elimination, and thus it will be important to consider implementation costs as they relate to other efficacy criteria within the TPP. The 50\% reduction in clinical incidence should therefore be considered a notional indicator, to be assessed in the context of prevailing epidemiological conditions, other available interventions, and costs, rather than a strict requirement.

Influence of transmission setting on efficacy. Participants recognized that local malaria transmission conditions will have a substantial influence on estimates of clinical

\footnotetext{
${ }^{4} \mathrm{EIR}$ is the number of infectious mosquito bites received by an individual in a single year.
} 
outcome. Therefore, one proposed approach for development of an investigational gene drive product is to think in terms of a first product that would be expected to meet the efficacy threshold in regions with low to moderate malaria transmis$\operatorname{sion}^{5}$ (although transmission would have to be high enough for an epidemiological impact to be measurable), and a second product that would be effective in regions of moderate to high transmission. The anticipated product claim could influence choice of sites for field testing; however, participants noted that trials conducted in areas of moderate transmission likely would be more straightforward to design and interpret.

Some workshop participants were concerned that the presence of multiple vector species in a specific locale would complicate interpretation of epidemiological efficacy results. Participants agreed on the importance of conducting initial small-scale field trials in regions and times of the year where the targeted mosquito species is a dominant malaria vector.

The feasibility of assessing the contribution of individual Anopheles species to malaria transmission in regions where multiple vectors are established was discussed. The group agreed that criteria for epidemiologic efficacy should apply to that proportion of malaria transmission that is attributable to the species that is targeted by the gene drive intervention, even though effective reduction of transmission by the dominant vector species also may lower transmission by other malaria vectors (Woolhouse et al. 1997). For example, in areas where A. gambiae s.s. and A. arabiensis coexist, but the former mediates most of the ongoing transmission, the potential disease impact of gene drive investigational products that suppress only populations of A. gambiae s.s. in laboratory studies would be modeled using baseline field data on proportions of all infective bites contributed by A. gambiae s.s. Planning could include assessment of human biting rate preintervention and postintervention.

Durability of impact. Gene drive-modified mosquito products are intended to reduce, and preferably eliminate, malaria transmission at the release site. Participants recognized that resistance to either the gene drive or the effector component may develop over time and that this could affect product efficacy. Product developers should utilize strategies to prolong the efficacy of the product, such as careful selection of guide RNA (gRNA) target sites that are highly conserved and intolerant of mutations (Kyrou et al. 2018), and techniques to reduce the rate of resistant allele generation, such as gRNA multiplexing (Marshall et al. 2017).

Product developers also should monitor for appearance of resistance during laboratory studies (Hammond and Galizi 2017). The group agreed that the gene drive mosquito product should have a minimal durability goal of health impact over 3 years, in alignment with the WHO standard that countries are considered to have achieved malaria elimination if they demonstrate no locally acquired malaria cases for 3 years (WHO 2018a).

It was suggested as a goal that the ideal gene drive mosquito product aim to remain efficacious (i.e., not disabled by

\footnotetext{
${ }^{5}$ The term high transmission has been used to indicate parasite prevalence $\geq 35 \%$ in children $2-9$ years of age, moderate transmission to indicate $10-35 \%$ parasite prevalence, and low transmission to indicate parasite prevalence $<10 \%$ (World Health Organization 2017e).
}

the appearance of resistance) for 10 years. It was accepted, however, that maintenance of the protective effect over this time might require occasional additional product releases in some circumstances. If resistance has developed during the intervening time, product variants having different gRNA target sites could be considered for potential re-release.

Other health benefits. Participants pointed out that in addition to contributing to an overall reduction in malaria incidence and prevalence, gene drive-modified mosquitoes have another potential benefit for malaria elimination and eradication. Gene drive mosquitoes also could contribute to preventing reintroduction of malaria in areas that have achieved elimination. This benefit would be dependent on the duration of the product's effect, which might differ between population replacement and population suppression strategies.

\section{Entomological efficacy}

Relationship between entomological and epidemiological outcome. Workshop participants considered how the goals for epidemiologic outcome relate to expectations for entomological impact. Given that the development pathway for gene drive mosquitoes proposes small-scale releases to test for entomological impact before larger releases focused on measuring disease impact (WHO 2014a, James et al. 2018), it will be important to set targets for entomological impact that would be likely to result in the desired reduction in clinical incidence in later trials.

Vectorial capacity. The criteria for assessing entomological outcomes were then debated. Entomological surveillance indicators relevant to malaria transmission have been described by WHO (2018b) and other specific endpoints may be defined to assess the efficacy of particular gene drive strategies. For example, these might include parasite infection rates in mosquitoes of the target vector species for replacement drives or proportions of the target species in the local Anopheles population for suppression drives, vector densities and survival, or EIR.

These direct entomological measures are difficult to relate back to desired epidemiological outcome, however. For this purpose, vectorial capacity ${ }^{6}$ may be an appropriate metric. Although vectorial capacity is difficult to measure directly (Dye 1986), it can be estimated from human metrics given knowledge of other interventions (in particular, access to treatment) (WHO 2015). The relationship between vectorial capacity, EIR, parasite prevalence, and the clinical incidence of malaria is captured by mathematical models (Griffin et al. 2010, 2014, Penny et al. 2016). In keeping with the prior discussion around establishing a minimal goal for epidemiological outcome, a $50 \%$ reduction in clinical malaria incidence would roughly correspond to a $65-90 \%$ reduction in vectorial capacity in one such model, with higher reductions necessary at higher levels of transmission, given the

\footnotetext{
${ }^{6}$ Vectorial capacity describes the number of new infections that the population of a given vector would induce per case per day at a given place and time, assuming that the human population is and remains fully susceptible to malaria (World Health Organization 2016c).
} 
nonlinear relationship between EIR and disease incidence (Ghani, pers. comm.).

Rate of spread. The participants also discussed how rapidly a product might reach entomological and epidemiological endpoints over a specified area. The rate at which these endpoints are reached will depend on the density at which the product is initially delivered, the size of the specified area, and the density of the local population of targeted mosquitoes, in addition to characteristics such as homing rate (gene conversion rate) and rate of reversion to resistance as described below. Products can be envisioned in which the effect is manifested in either heterozygous or homozygous gene drive-containing mosquitoes. There was no resolution on a general goal for how quickly the effect must be achieved, as it is expected to vary on a case-by-case basis, but it was pointed out that the financial cost implications must be taken into account when designing the delivery strategy.

\section{Considerations for efficacy testing}

Efficacy parameters. The group was challenged to think specifically about what parameters should be measured under containment in the laboratory, insectary, or indoor cage facility as key predictors of efficacy in the field, with the understanding that there will be some important differences between population suppression and population replacement gene drive strategies. Participants advised that some parameters that easily can be measured in containment probably are not particularly relevant predictors of efficacy, while some key predictors of entomologic efficacy cannot realistically be measured outside of the field setting. Therefore, the charge was to focus on those parameters that could be assessed in containment, which would provide useful criteria for selection of investigational products to move into field testing.

It was emphasized that thought must be given early in development to molecular characterization of the gene drivemodified mosquito investigational product, both for quality management and regulatory purposes. This might include description of nuclease tissue specificity and expression level, sequence insertion and flanking regions, absence of plasmid backbone sequence, and copy number. The desirability of keeping the transgenic construct as short as possible and without any bacterial sequences (other than the Cas nuclease) was raised.

\section{Construct-specific parameters}

Homing rate. Homing rate was considered to be a key parameter for predicting rate of spread of the gene drive construct. All viable investigational products must demonstrate a high homing rate to be considered for advancement, but the homing rate necessary for success will be influenced by fitness effect and this interaction must be considered in setting a minimum requirement. One suggestion for measuring homing rate was to assess deviation from Mendelian expectations in $\mathrm{F} 1$ progeny from single-generation crosses of individuals heterozygous for the gene drive construct with the wild-type comparator.

The homing rate can change over multiple generations and may be influenced by the genetic background, particularly as it concerns homing-resistant variants of target sequences. Well-controlled experiments will take care to distinguish homing rate from any skewing of transmission patterns due to other factors associated with the genotype. To obtain an accurate rate of homing, it will be important to follow the effect at the target sequence.

Site and level of transgene expression. Genomic location of the construct can affect expression. Leaky somatic expression has been noted as a problem affecting fitness and reproductive success (Gantz et al. 2015, Hammond et al. 2017). Parental deposition of the endonuclease can be a cause of mutation in the fertilized zygote or embryo, resulting in reduced homing and fecundity (Champer et al. 2017). Parental deposition or leaky somatic expression of the endonuclease can be difficult to determine accurately without intricate crossing schemes. In some cases, phenotypic mosaicism can serve as a proxy for these events. Use of a promoter that tightly restricts nuclease expression to the germline likely will improve fitness and delay emergence of resistance (Hammond et al. 2018).

Off-target effects. Off-target effects of the endonuclease used in the construct (i.e., activity at genomic sites other than the intended target site) may be more important for population replacement strategies where the transgenic construct must remain present at high frequency in the target population over long periods of time. Off-target effects may decrease fitness, influencing characteristics such as survival, mating success, and fecundity, and thereby compromise entomological and epidemiological outcomes. It was discussed, however, that off-target effects are likely to be very heterogeneous at the individual level and nonclonal, meaning any off-target signature would be different in each mosquito. Moreover, the frequency with which off-target events occur is a matter of some debate and is likely to be gRNA specific.

Individually, off-target effects are unlikely to have a major impact at the population level, although collectively their accumulation may influence outcome. Notably, because progeny with decreased fitness are less likely to mate and spread the transgenic construct effectively, such modifications are more likely to be lost due to natural selection. Bioinformatics may help to identify sequences that are potential sites for off-target activity. However, the group agreed that the most practical way to evaluate off-target effects is by following fitness and other phenotypic changes rather by than trying to detect a very low frequency event by genome sequencing, especially since natural population heterogeneity would complicate such an analysis. They also noted that new endonuclease enzymes with increased sequence fidelity are being developed for human therapeutic applications (e.g., Kleinstiver et al. 2016), which also could be tested for development of gene drive-modified mosquitoes.

Nonhomologous end-joining. It was agreed that nonhomologous end-joining is only one of multiple alternatives to homologous repair that might impact efficacy and promote the development of resistance. These mechanisms should be considered collectively when evaluating potential for resistance to develop against the construct. Prior studies have addressed these events (Hammond et al. 2016); although given the important role of resistance in the success of gene drive-modified mosquito products, they warrant further study. 
Construct-induced phenotypes at the individual level. Vector competence, which is a major element of vectorial capacity, can be assessed in the laboratory. For both population replacement and suppression strategies, risk assessment likely will require determination that the transgenic construct does not increase vector competence in comparison to wildtype mosquitoes of the same genetic background (see Considerations for biosafety testing, Vector competence section). For population replacement strategies intended to make mosquitoes refractory to development of the pathogen, a critical efficacy criterion will be demonstration of substantial and sustained reduction in carriage of Plasmodium parasites. Methods are available for conduct of both membrane feeding and direct feeding assays to measure parasite transmission blocking (Miura et al. 2013, Coulibaly et al. 2017). The group noted, however, that standardized protocols are needed for reproducibly assessing vector competence and that these tests should be adequately replicated to provide statistical power.

Other parameters important for population replacement include changes in parasite extrinsic incubation period or other factors that might signal potential for increased virulence in the human host. As discussed further below, the group recommended that developers of gene drive-modified mosquitoes for population replacement test their efficacy against multiple parasite isolates or species to provide a better prediction of field performance.

General life history parameters. Suggested life history parameters included the following: adult longevity; mortality rate; egg numbers; egg hatching rate; larval-pupal development time; larval-to-adult survival; sex ratio of progeny; adult biting rate and host preference; and adult mating competitiveness, including behaviors relevant for mating such as swarming. While all of these parameters were recognized as contributing to the entomological and epidemiological outcome, discussion led the group to consider that it would be more practical to concentrate initially on an empirical assessment of the ability of the construct to drive through a population. If the investigational gene drive product is not as effective as expected, then developers may wish to examine these other factors more systematically to determine where the flaw lies, beginning with those parameters identified by modeling as having the most impact on driving behavior, or they simply may choose to eliminate the investigational product from further consideration. If the developer chooses to proceed with measurement of these parameters, some may need to be tested under a variety of nutritional and environmental conditions to get a better sense of how the candidate might perform under field conditions.

Efficacy at the population level. Functionality of a population suppression strategy can be measured in containment by following population decline in cage studies. For population replacement, there was no consensus on how competence for malaria transmission should be measured on a population level, but possibilities include evaluation of the kinetics of transgene spread and maximum transgene frequency, parasite infection levels and stability of the antiparasitic effect in laboratory cage populations over multiple generations, and modeling utilizing these data will play an important role in predicting potential population-level impact.
Resistance development. As mentioned above, rapid development of resistance to the drive or effector mechanism would limit the efficacy of an investigational gene drive product. Loss of efficacy could develop due to mutations in the transgenic construct itself, and in strategies aimed at coupling an effector cargo to the gene drive, this could lead to loss of the cargo. However, perhaps a greater concern, relevant to both suppression and replacement strategies, will be mutations in the target sequence in the mosquito genome that render the site no longer cleavable by the RNA-guided endonuclease (Unckless et al. 2017). This target site resistance can result from pre-existing standing variation in the population or can be induced by the nuclease activity itself, where repair by end-joining or imprecise/incomplete homologydirected repair can produce variant, noncleavable alleles.

The group agreed that observation of functional resistance (defined as nuclease-resistant alleles that restore some function to the target gene) that is strongly selected for in the context of contained studies would trigger a critical decision as to whether to advance the investigational product, given that the opportunities for resistance to develop will be even greater under field conditions where larger populations and greater genetic diversity are involved.

Influence of resistance on gene drive function. For population suppression strategies, evidence in the laboratory for development of resistance alleles that preserve the function of the target gene should preclude further advancement of the investigational product, at least until such time that the investigational gene drive can be redesigned to overcome such resistance alleles. Accumulation of resistance alleles that do not restore target gene function, which may result in some fitness reduction, may also be expected, but would not necessarily disqualify an investigational product.

For population replacement strategies, in addition to alleles that produce cleavage-resistant target sites, another problematic class of alleles would be those leading to a loss of linkage between the drive and effector mechanism (Beaghton et al. 2017). If resistance alleles form at rates observable in laboratory cage studies, the decision to advance the investigational product should be informed by a model-based assessment that accounts for both their formation rate and fitness consequences to estimate how long the drive/effector could persist at a sufficiently high level to provide the desired level of protection against disease. At a minimum, any functional mutation should have no fitness advantage over the gene drive construct. With population replacement strategies, there also is the possibility of selection for development of resistance by the parasite to the effector mechanism, which, as discussed below, must be accounted for in design of the effector mechanism.

Detecting resistance to the drive in target mosquitoes. Selective pressure for emergence of endonuclease resistance due to existing variation in target sequences is expected to be greatest for population suppression strategies (Marshall et al. 2017). The frequency of natural variation at the target locus can be examined by genome sequencing of mosquitoes collected from the field, acknowledging that (1) rare genetic variants will be difficult to detect and (2) genomic sequence data for projects with a large intended geographic range may not be as comprehensive as desired. Opportunities for resistance to arise as a result of random mutations are likely to be 
greater for population replacement strategies since they will need to function over long periods of time.

While other options are possible, one methodology suggested by participants to test for functional resistance is to conduct many crosses of heterozygous gene drive-modified and wild-type mosquitoes. The power to detect rare resistant alleles will depend on the number of independent crosses and the expected probability of resistance developing per cross. A structured approach to choosing the minimum number of crosses would specify the required probability, or confidence level, for detecting resistance.

Looking at the sequence of the gene drive target site in any progeny that does not acquire the modification will identify nuclease-induced mutations, but would not determine whether they are functionally resistant. Therefore, resistant progeny should be tested again in a 50:50 cross with gene drive-modified mosquitoes to determine whether the mutation preserves the function of the target gene and impedes the function of the drive. These data could help to inform modeling to estimate the probability that development of a functionally resistant allele will be missed in cage trials. Conduct of resistance testing under selection pressure will give the best chance to functionally characterize mutations.

Random target site mutations will occur rarely and, while it will be important to look for their appearance over time in gene drive-modified mosquito strains, they may be difficult to observe in contained studies of small populations. As an alternative, the group discussed the idea of deliberately mutagenizing the target site in the laboratory to test the sensitivity of the gene drive to target site variation and what effect this has on fitness. For example, as a first step, DNA from the mutagenized target site could be incubated with the endonuclease-gRNA construct to test whether the mutagenized site still could be recognized and cut.

Detecting parasite resistance to population replacement strategies. Parasite resistance to the effector mechanism(s) was identified as a significant concern for population replacement strategies. It was agreed that this will be difficult to measure until numbers of resistant parasites grow to levels that might be distinguishable by genomic or phenotypic characteristics, which is unlikely to occur until after field releases. However, due diligence in containment will include testing for vector competence using parasite samples from different locations, including, but not limited to, those circulating at the field site, to check the breadth of infection blocking efficacy.

It was recognized that outcomes for population replacement may depend upon the number of parasites with which the gene drive mosquitoes will be infected, and that breakthrough is more likely to be seen with a higher parasite burden. Since breakthrough events may relate to selection of resistant parasites, decision on the appropriate challenge dose for vector competence testing in containment is critical. For purposes of candidate downselection, this should be tested with numbers higher than the anticipated transmission level at the field site.

The best defense against selection of parasites that are resistant to the effector mechanism is to incorporate multiple effector systems against different parasite targets into the investigational gene drive product to create redundant pathways for protection, although it must be determined whether such multiplexing techniques introduce fitness effects or other complications. Reduction of the parasite population through use of additional malaria control tools can assist in decreasing opportunities for breakthrough events to occur.

\section{Suggested order of testing for selection of viable investigational products}

Investigational products that should not be advanced can most efficiently be identified by beginning with studies that take the least amount of experimental effort. Participants were challenged to propose a study progression that would facilitate early elimination of nonperforming candidates, following initial confirmation of biased inheritance due to homing. It should be noted that alternative scenarios are feasible, so these suggestions should be taken as illustrative only.

Cage studies should be replicated until there is sufficient statistical power to derive unambiguous conclusions. Consideration should be given to the relative merits of overlapping versus discrete generations in design of cage trials, because overlapping generation experiments can provide useful data on fitness effects, while discrete generation experiments provide a more precise window into the inheritance-biasing effects.

\section{Population suppression products}

1. Evaluate the efficacy of the suppression phenotype, based on the homing rate (gene conversion efficiency) and viable genotypes of the progeny. The suggested methodology is to carry out single-generation crosses (heterozygous transgenic males crossed with homozygous wild-type females, and the reciprocal) and measure rates of inheritance of a scorable drive allele marker (e.g., fluorescent marker). A performing construct will display biased non-Mendelian inheritance of the transgenic construct in the F1 progeny. It could be useful to test for development of functional resistance at this point, before proceeding to cage studies, since any construct succumbing to functional resistance should be eliminated from further advancement.

2. Fitness testing of heterozygous and homozygous males and females, using standard published assays for lifetable parameters such as male fitness, mating efficiency, fecundity, and blood feeding rate (Moreira et al. 2004, Kyrou et al. 2018).

3. Performance in small cage studies, by introducing gene drive-modified mosquitoes in combination with the wildtype comparator strain and comparing the level of population suppression with the prediction from modeling based on previously measured parameters (homing rate, fecundity, etc.). The efficacy endpoint in cage trials will be ongoing population suppression or disappearance.

\section{Population replacement products}

1. Evaluate the efficacy of the effector construct for blocking of Plasmodium falciparum infection. The proposed methodology is to challenge the gene drivemodified strain (whether to use the homozygous or heterozygous strain may depend on the product goal) with cultured $P$. falciparum gametocytes at two parasite concentrations, comparing parasite development to that in the unmodified mosquito strain of the same genetic background. Suggested parasite concentrations 
are a high-intensity infection of average $\sim 50$ oocysts, and low-intensity infection of average $\sim 5$ oocysts, which is more reflective of natural infection levels. The endpoint is sporozoite numbers in salivary glands. Some participants proposed that a candidate that does not completely block salivary gland infection under low-intensity conditions should not be selected for advancement. Others suggested that the predicted epidemiological impact of a less than perfect reduction in sporozoite load should be considered before ruling out a candidate, taking into account the potential for development of resistance by the parasite as discussed above.

2. Measure effector spread in cage populations. The suggested methodology is to introduce the driving strain in a cage with the wild-type strain, and measure frequency of marker phenotypes representing presence of the drive allele over time (as well as marker phenotypes representing some varieties of resistance alleles where possible). As proposed above, inheritance of the gene drive construct can be followed using a scorable drive allele marker. A viable construct should increase in allele frequency or at least persist in the cage. This assay will identify a construct with a fitness load that retards the driving mechanism, without the need for detailed fitness assays. Cage studies should measure time until all wild type alleles are gone (or all individuals have acquired the gene drive construct in at least one copy, for example as measured by a fluorescent marker). It is recommended to continue the study for several additional generations after this observation to assess for appearance of resistant alleles. The power to detect rare resistant alleles will depend upon the number of independent crosses occurring in the cages, and therefore the number of mosquitoes in the cages.

3. For those candidates that perform well in testing for effector fitness cost, as inferred by invasion dynamics compared to modeled estimates, detailed measurement of additional fitness parameters would include longevity, development rate, and reproductive parameters such as mating efficiency, fecundity, and blood feeding rate (Dong et al. 2011).

\section{Influences on efficacy testing}

The group agreed that conditions under which efficacy testing is conducted may influence the comparability of data as well as relevance of data to expected field performance.

Genetic background. The genetic background may influence results of efficacy testing. The group agreed with prior recommendations on the desirability of conducting tests to obtain data for the regulatory dossier in a genetic background as similar as possible to that of local mosquitoes found at the site(s) of proposed release (WHO 2014a, James et al. 2018). This can be done by backcrossing the transgenic construct into mosquitoes from a colony derived from locally collected wild-type mosquitoes or by directly transforming mosquitoes from the local colony with the gene drive DNA construct. If release will occur at multiple sites, but these sites are connected geographically or otherwise not reproductively isolated, a single colony derived from locally collected mosquitoes should suffice. If the release sites are distant, one will need to establish models of transgene spread to determine whether additional local colonies might be needed.

Local entomologists should be supported to colonize the target species as part of early collaborations. Participants discussed the concern that it is not always straightforward to colonize local mosquitoes, and therefore that the ability to colonize the local target mosquito species is an issue to be addressed in early stages of development, including in selecting a site for field testing. They also acknowledged that bottlenecks induced by the small population size maintained in physical containment gradually will lead to loss of genetic variability as well as selection of traits amenable to laboratory rearing, so that newer colonies will be most relevant to wild-type mosquitoes (Aguilar et al. 2005, Ng'habi et al. 2015).

In situations where access to a colony of local mosquitoes is not available, such as early testing of gene drive constructs in discovery laboratories, the group considered the possible utility of testing for efficacy in a panel of different mosquito lines, including A. gambiae and also A. coluzzii, if field testing will occur at a site were these two sibling species might be likely to interbreed. Some cautioned, however, that care should be taken not to introduce non-native species into field sites.

Parasite strains. Efficacy of a population replacement strategy, where the effector mechanism is directed against Plasmodium cell surface or other targets, may be influenced by the parasite strain in which it is tested, since many Plasmodium antigens are known to exhibit sequence variation. Therefore, efficacy testing for population replacement candidates initially should be conducted against a panel of $P$. falciparum lines to assess for variability in results that would provide a signal to eliminate the investigational product from further advancement. Participants proposed that it would be desirable for the infection blocking competence of a gene drive mosquito candidate to be validated in an independent laboratory before it is considered for advancement to field testing.

Before release of a population replacement gene drive investigational product, efficacy testing in containment should be repeated with parasites circulating locally at the release site. This might be accomplished by using a mobile containment facility or by transporting gametocyte-carrying patients to the laboratory. Initial field testing of the activity of the effector construct against local parasites using a nondriving or self-limiting version of the investigational product should be considered a risk management strategy.

Environmental effects. Dynamic conditions, such as temperature, humidity, larval and adult density, source of blood meal, and release ratio, can affect results of testing in physical confinement. While recognizing this, participants expressed doubts about the extent to which this can be adequately explored in early studies and did not propose any specific recommendation on standardized conditions for conducting cage trials. However, these parameters should be recorded in all studies as part of good laboratory practice.

Experiments carried out inside large cages or semifield mesocosms to assess the mating capabilities of transgenic mosquitoes can reveal effects on the fitness/vigor/behavior of 
the mosquitoes that are difficult to observe in smaller cages (Facchinelli et al. 2013, 2015, Aldersley et al. 2019). This information could contribute to decision-making about whether or not to advance an investigational gene drive product to field testing. However, it is widely acknowledged that behavior in semifield testing may not strictly reflect future field performance, and therefore, the decision as to whether such testing is an essential step in the development pathway for a particular investigational product should be made by developers and regulators (James et al. 2018).

\section{Considerations for biosafety testing}

Those investigational gene drive products that meet efficacy qualifications must also be subjected to safety testing. Pertinent broad protection goals have been identified as human and animal health and biodiversity, and possibly water quality (Roberts et al. 2017, Teem et al. 2019). The go/no-go safety criterion for moving a gene drive-modified investigational product to field testing has been proposed as "will do no more harm to human health than wild-type mosquitoes of the same genetic background and no more harm to the ecosystem than other conventional vector control interventions", (James et al. 2018). Thus, the appropriate comparator for adverse effects could be either unmodified A. gambiae mosquitoes or insecticides; the latter may be especially relevant for population suppression products. In this case, the focus would be on adulticides and larvicides that are used locally for malaria vector control.

For collection of experimental data, it is important to know whether a validated assay method already exists and to use it if available. Experimental studies should be designed specifically to test the effect of the gene drive construct. For some types of studies, the test substance will be the gene drive-modified mosquito and these should be done in comparison to nonmodified mosquitoes of the same genetic background. In this case, a decision must be made as to whether heterozygous or homozygous gene drive-modified mosquitoes are the most relevant test material. For other studies, such as toxicity testing, the appropriate test substance may be the recombinant protein produced by the transgenic construct, if sufficient amounts can be produced.

Vector competence. Vector competence studies respond to concerns about human and animal health. The question of whether the gene drive modification could result in increased competence for transmission of malaria or other mosquitoborne pathogens is expected to arise in risk assessment (Roberts et al. 2017). Testing of vector competence for Plasmodium species was addressed above. It will be important to provide a credible basis for the choice of other pathogens which will be tested in vector competence assays. The methodology utilized by WHO for prioritizing emerging pathogens for actions to prevent epidemics (WHO 2017c) might be adopted for this purpose.

For safety studies, consideration also should be given to other pathogens known to be transmitted by A. gambiae, such as o'nyong'nyong virus and filariasis. Vector competence for o'nyong'nyong can be assessed by standard membrane feeding assays (Vanlandingham et al. 2005, Pike and Dimopoulos 2018). However, determining vector competence of A. gambiae for Wuchereria bancrofti, the parasite most responsible for lymphatic filariasis in Africa, using membrane feeding is not likely to be possible because of the lack of an efficient laboratory model for infection and the increasing difficulty of obtaining wild isolates during nationwide mass drug administration campaigns. In this case, insights into the potential for increased transmission can be gained by testing mosquito responses to intrathoracic injections of microfilariae of a related parasite, Brugia malayi (Erickson et al. 2009), and by examining specific anatomical characteristics that influence transmission (McGreevy et al. 1978, Amuzu et al. 2010). Any potential for increased rates of development and transmission in the mosquito must also be balanced by the significant attrition of filarial worms in humans (Irvine et al. 2015) and the mediating effects of vector control and mass drug administration.

Allergenicity and toxicity. Allergenicity and toxicity studies respond to concerns about human and animal health as well as water quality (since mosquito larvae breed in water). The potential for increased toxicity or allergenicity resulting from the gene drive modification has been raised as a hazard (Roberts et al. 2017, Teem et al. 2019). Several participants asserted that allergenicity would only be a relevant hazard if the transgenic proteins are expressed in the mosquito salivary glands. Others suggested that an effort also might be made to determine whether expression of other known allergenic proteins in the salivary glands is dysregulated as a result of the modification.

If toxicity effects are to be examined directly in the laboratory, it will be important to conduct risk assessment to prioritize nontarget species for which there is greatest potential for interaction with the modified mosquitoes (Raybould 2007); examples might include a mosquito predator or organism that occupies the same ecological niche. There is well-documented guidance in the Codex Alimentarius (Food and Agriculture Organization, CAC/GL 45-2003 and CAC/GL 68-2008) for testing toxicity and allergenicity of genetically modified foods that may be adapted for gene drive-modified mosquitoes, although the most relevant route of exposure would change from through ingestion to through mosquito bite. Some information on direct effects of fluorescent marker proteins is already available in the literature (Richards et al. 2003, Pioneer Hi-Bred International 2011, Food Allergy Research and Resource Program 2017).

Insecticide resistance. The gene drive-modified investigational product should be no more resistant than the local comparator mosquito to insecticides that are available at the testing site (James et al. 2018). WHO has published test procedures for insecticide resistance (WHO 2016a).

Spread of the gene drive construct to other organisms. Consideration of adverse effects to nontarget organisms arising from vertical or horizontal gene transfer relates to biodiversity hazards. Participants remarked on the importance of considering what potential harm could arise from movement of the construct to other organisms, rather than considering it to be a harm in itself.

The rate of spread of the gene drive construct to other species will be determined by the rate of hybridization between reproductively compatible members of the A. gambiae species complex (Lee et al. 2013, Fontaine et al. 2015). The 
intent of the investigational product should be taken into account in considering the risk of vertical gene transfer. If the intent is for the modification to spread to other reproductively compatible members of the A. gambiae species complex, most of which also are malaria vectors, then it might be reasonable to consider these together as a single target species. If not, then other members of the complex outside A. gambiae s.s. must be considered nontarget organisms.

In considering potential for horizontal gene transfer, participants pointed out that naturally occurring homing endonucleases have proven unsuccessful in transferring to animals with a segregated germline, in comparison to other selfish elements such as transposons (Burt and Trivers 2006). Participants suggested there is no obvious reason to suspect that engineered gene drives based on the same mechanism of action as naturally occurring homing endonucleases, which is the case for RNA-guided endonucleases, would be more likely to transfer to unrelated organisms. Moreover, a prior problem formulation exercise for gene drive-modified mosquitoes conducted by a multidisciplinary group of experts concluded that horizontal gene transfer is not likely to occur to other organisms on any relevant time scale and is not a pertinent pathway to harm (Roberts et al. 2017).

Nonetheless, from a precautionary perspective, the potential for horizontal gene transfer is expected to be considered on a case-by-case basis in risk assessment. One proposed method to assess for the possibility of spread of the gene drive construct by homing is to examine the genome of the nontarget species of interest, where genome sequence is available, for presence of target site sequence that would be recognized by the gene drive construct. This should account for sequence variation known to be tolerated for homing to occur in the target species. In their initial research, developers may wish to take into consideration the presence of chosen target sequences in species that closely interact with A. gambiae.

Niche replacement. Niche replacement-the possibility that another undesirable organism may invade the ecological niche vacated by reduction or elimination of A. gambiaeoften is raised as a concern for both health and biodiversity. It should be noted in this regard that gene drive-modified mosquitoes are being developed in the context of integrated vector management, where other vector control interventions are likely to affect additional species.

Given the risk hypothesis "will do no more harm to human health than wild-type mosquitoes of the same genetic background and no more harm to the ecosystem than other conventional vector control interventions" (James et al. 2018), the appropriate comparator for consideration of niche replacement may be insecticides (adulticides and larvicides) that are used locally for malaria vector control. It was pointed out that current insecticides are broad spectrum and will have a range of negative effects, including reductions in the size of other invertebrate populations as well as fish and other vertebrate populations. Gene drive mosquito investigational products are expected to have much more restricted effects.

Whether insecticides allow for realistic examination of niche replacement effects is undetermined. However, it was noted that intensive application of current vector control tools has resulted in proportionate changes in A. gambiae sibling species composition, with decreased representation by species that primarily rest and feed indoors (Kitau et al. 2012).
Whether this proportionate change is associated with an increase in absolute numbers of other species, which would be the relevant hazard, is unclear because most studies to date have not examined mosquito numbers. In one study which did, there was no evidence for an increase in absolute numbers of A. arabiensis when A. gambiae s.s. abundance decreased as a result of bed net use (Bayoh et al. 2010). However, an older study reported that reduction in another important African malaria vector, A. funestus, which is very anthropophilic, resulted in an increase in A. rivulorum, which is a possible, but certainly much less prominent malaria vector (Gillies and Smith 1960). Thus, risk assessment may also need to consider what risks are associated with species most likely to fill the niche vacancy in comparison to risks posed by the targeted mosquito species.

Other adverse effects on biodiversity. The group discussed potential for adverse effects of adult gene drivemodified mosquitoes on species living in terrestrial habitats (e.g., predatory invertebrates, beneficial invertebrates, plants, and vertebrates) as well as of larval gene drive-modified mosquitoes on species living in aquatic habitats (invertebrates, vertebrates, and decomposers). They were unable to identify a scenario they considered likely. A recent review of evidence for negative effects of decreased A. gambiae density on potential predator species found no evidence to suggest it is a critical food source (Collins et al. 2019). Participants also discussed the potential adverse effect of loss of mosquitoes as pollinators, but noted there is no evidence that A. gambiae plays an important role in pollination.

In considering possible adverse effects of population suppression gene drive products, it should be pointed out that the goal of these strategies is to reduce mosquito numbers sufficiently to break the transmission cycle, and recent modeling results based on conditions in Burkina Faso suggest that global eradication of A. gambiae by such strategies is not possible, although local elimination may occur under certain conditions (North et al. 2019). Again, however, this is a question that should be addressed on a case-by-case basis in risk assessment.

Genotypic and/or phenotypic stability. Developers should continue to monitor stability of the insertion over multiple noncontiguous generations in the laboratory, as this will be a point of interest for regulators. The group discussed the need for a phenotypic durability plan, capable of demonstrating that the product that has received regulatory approval remains consistent after release. This will require definition of regulatory expectations as well as robust and reliable detection methods.

Behavioral changes. Possibilities that the gene drive modification might change mating, host seeking, or feeding behaviors, or cause some change that would result in expanded geographic range (such as a change in temperature sensitivity) were considered. However, participants noted that available evidence from population suppression by insecticides does not indicate a plausible pathway by which mosquitoes could acquire benefit from gene drive modification.

Change in feeding behavior can be assessed in the laboratory by comparing the probing and biting rate of the gene drivemodified mosquitoes versus that of the relevant unmodified 
comparator strain. This would be facilitated by well-validated protocols for such studies. While unlikely, the possibility that changes in feeding time or endophagy/exophagy should at least be considered and, perhaps, monitored postrelease.

Efficacy studies carried out in containment at different temperatures (Environmental Effects section) may provide insights into any potential for a change in geographic range of the gene drive-modified mosquitoes. Postrelease monitoring is expected to include examination of the range of gene drivemodified mosquitoes.

\section{Considerations for collection of field data}

Information about the receiving environment for release of investigational gene drive products will be required for risk assessment and regulatory dossiers. Moreover, certain field data will contribute to modeling and design of relevant cage trials, modeling field performance of constructs to predict efficacy and safety, and planning of release and monitoring strategies. These data include information on size of the target mosquito population, survival rate, movement and dispersal of the target species, and responses of these variables to seasonal environmental factors such as temperature and rainfall, population structure and gene flow, landscape in the receiving environment (potential barriers to gene flow), intrinsic rate of reproduction, distribution of breeding sites, factors influencing persistence of mosquitoes during the dry season, and impact of larval competition on population density. Other field data need for regulatory applications will include information on insecticide usage and susceptibility at the field site, as well as information on the local ecosystem. Modeling of epidemiological outcomes will require information on malaria incidence, prevalence, and treatment coverage with antimalarial drugs.

Sampling methods. Collection of baseline data at potential field sites should begin early and be conducted over at least 2 years (James et al. 2018). Sampling methods should be designed to obtain the most realistic representation of changes in the population, such as variation in density and mix of species present. Currently available trapping methods for adult mosquitoes can introduce bias (James et al. 2014), so larval sampling also was considered necessary. Sampling mosquitoes at aquatic stages will yield multiple species, including those that rarely go near or into homes, and are less likely to be captured by common adult sampling traps. Such comprehensive sampling requires prior knowledge of mosquito habitats and is labor intensive. The sampling scheme will be dependent upon site characteristics, but should take seasonal variation into account. The sampling sites ideally would include areas of high and low malaria transmission.

Prediction of eventual spread of the gene drive construct over larger areas will require modeling and data that can inform these models on the scales of interest. National malaria control or vector control programs may conduct vector surveillance at sentinel sites (WHO 2017d) that could provide information across broader geographic areas and regions of differing malaria transmission intensity if these data can be accessed.

Population data. The group recommended that information on potential movement of gene drive-modified mos- quitoes can be derived most efficiently from large-scale genetic screening, with assessment of populations collected from multiple sites. Genome sequencing also can provide insights into local population structure of the target species, other species present, and rates of hybridization among sibling species. Longitudinal sampling and genome sequencing in a region where $A$. gambiae repopulate after the dry season could provide information on the influence of aestivation and aquatic refugia.

Genetic data will provide insights into effective population size and levels of containment within a population. This can take advantage of concepts such as geogenetic mapping (Bradburd et al. 2016). Subpopulations that exhibit some degree of genetic isolation could limit the spread of the gene drive construct, and understanding of fine spatial structure may be especially important for predicting success of gene drive-modified mosquito products. This may require intensive temporal sampling in potential target areas.

The traditional method of assessing mosquito dispersal is by mark-release-recapture (MRR) studies (Benedict et al. 2018). As a complement to genome sequencing, traditional population census and MRR studies can provide useful population data. MRR may be helpful for assessing dispersal at the field site, which would contribute to design of a release strategy. However, genomic data will provide better information on long-distance dispersal and the spread of introduced transgenes. Methods for estimating dispersal within and across 1-3 generations have now been developed for mosquitoes (Jasper et al. 2019). Participants concluded that genetic analysis will provide a better approach to estimate gene flow and migration over intermediate to long distances and durations, while MRR studies can provide unique insights for short distances and durations.

Potential ecosystem interactions. National biodiversity action plans (Convention on Biological Diversity 2019) and published lists of threatened species (International Union for Conservation of Nature and Natural Resources 2019) should be consulted to determine whether any charismatic or valued species are found in habitats at or near the field site that should be considered in risk assessment. It will be important to prioritize nontarget species for which there is greatest potential for interaction, which can be determined by risk assessment (James et al. 2018). Baseline ecological studies should include information on other species that are found in larval breeding sites and species that perform similar ecological functions as A. gambiae (ecological guilds). In considering potential ecosystem effects, thought also should be given to any possible beneficial effect for biodiversity, such as reduction in insecticide exposure.

\section{Conclusions and Recommendations}

A recent study on the development pathway of gene drivemodified mosquitoes noted the critical nature of the decision to move from contained (physically confined) testing in a laboratory, insectary, or indoor cage facility to semifield or field testing, given the possibility that the modification might become established in the local target mosquito species as a result of the release of only a small number of gene drivemodified mosquitoes (James et al. 2018). This study further recommended that "candidates considered for movement to 
field testing should be based on a sound hypothesis and demonstrate efficacy and fitness characteristics in the laboratory that are consistent with the TPP and anticipated product claim."

As developers conduct studies to identify viable investigational gene drive products, their work will transition from discovery research to product development. PPCs identify a core set of attributes for a general product type and are useful to provide early guidance for development of new products (e.g., WHO 2014b). TPPs are planning tools that identify the desired attributes of a particular product for a particular indication (e.g., Burrows et al. 2017; Drugs for Neglected Diseases Initiative). The availability of PPCs and TPPs with well-considered criteria can facilitate the product development process by focusing time and resources on investigational products most likely to have the desired effect. Possible TPP parameters for gene drive-modified mosquitoes intended for population replacement previously have been proposed (Carballar-Lejarazu and James 2018), but there currently is no widely accepted guidance for what constitutes an acceptable product.

Proposed criteria for PPCs of gene drive mosquitoes are summarized in Table 1. Some workshop participants were concerned that it might be challenging to develop a single set of PPCs for low-threshold gene drive mosquito products because expectations for efficacy may differ across the wide variety of malaria transmission settings in Africa. However, most agreed that because gene drive-modified mosquitoes are being proposed as a public health tool, it is desirable to identify overall public health efficacy objectives, which researchers should keep in mind throughout the various stages of product development and testing. The majority of participants in the first workshop ${ }^{1}$ agreed that an epidemiological efficacy goal, based on predicted reduction in malaria incidence, should be considered in PPCs and TPPs for gene drive-modified A. gambiae. It was understood that the case for achieving that goal would have to be based on modeling until the time that large trials to directly test epidemiological impact can be conducted. The workshop participants emphasized the importance of accurate modeling to estimate the potential efficacy of gene drive candidates and contribute to decision-making about moving to field testing, but noted the need for additional data to refine current models.

TPP efficacy parameters for gene drive-modified mosquitoes are proposed in Table 2. It has been recommended

Table 1. Proposed Criteria for Preferred Product Characteristics of Gene Drive-Modified Mosquitoes

1. Indication-target vector and parasite species

2. Epidemiological efficacy goal-reduction in clinical incidence of malaria

3. Entomological efficacy goal-reduction in vectorial capacity commensurate with epidemiological protection goal

4. Duration of protection-time over which the epidemiological efficacy impact will be evident

5. Time to impact - time required for the product to achieve epidemiological and entomological goals over a specified area

6. Safety for human health and the environment
Table 2. Proposed efficacy Parameters for Target Product Profiles of Gene

Drive-Modified Mosquitoes

1. Homing rate-predictor of rate of spread and time to impact

a. Deviation from Mendelian expectation of inheritance in cages

2. Life history and reproductive success-predictor of rate of spread and time to impact

a. Adult longevity

b. Adult biting rate

c. Mating efficiency

d. Egg clutch size and hatching rate

e. Sex ratio of progeny

f. Development and mortality rate at different life stages

3. Construct functionality - predictor of entomological and epidemiological efficacy

a. Population suppression-population decline in cages

b. Population replacement-reduction in carriage of the target parasite species

4. Functional resistance-predictor of duration of protection

a. Population suppression and replacement-functional resistance to the drive

b. Population replacement-parasite resistance to the effector(s)

that an investigational gene drive-modified mosquito product should be expected to be at least as efficacious as current malaria control tools to support a decision to advance to field releases (James et al. 2018). Suggestions for the minimal level of predicted reduction in malaria incidence that would justify further development varied from $20 \%$ to $50 \%$, in the presence of other malaria control methods used according to standard of care. However, it was noted that the epidemiological goal should apply only to that proportion of disease that is transmitted by the mosquito species targeted by the investigational product, and that the reduction goal will be proportionate to the baseline infection rate at the field site, as this varies greatly across Africa.

The group further recommended a minimum goal of 3 years for durability of protection. There was no agreement regarding a minimal goal for time to impact, although "an epidemiologically relevant timeframe as needed to achieve disease control" has been the standard expectation (WHO 2014a).

A major challenge in developing a TPP for gene drivemodified mosquitoes is to translate the epidemiological efficacy goal into measurable entomological parameters on which to base the decision about whether a particular investigational product is suitable for advancement to small-scale field testing. Theoretically, vectorial capacity, which describes the capability for disease transmission by a vector to a host as influenced by a number of behavioral, ecological, and environmental factors (Brady et al. 2016), can be calculated based on data collected under contained conditions and translated into an approximate epidemiological outcome in the field based on understanding of malaria transmission dynamics at the setting of interest (Smith and McKenzie 2004).

With respect to safety criteria, participants in the second workshop ${ }^{2}$ endorsed a prior recommendation of the standard that gene drive-modified mosquitoes "will do no more harm to human health than wild-type mosquitoes of the same genetic background and no more harm to the ecosystem than 
other conventional vector control interventions" (James et al. 2018). This, however, raised the question of whether the most appropriate comparator for some aspects of risk assessment, particularly adverse effects on biodiversity, should be the insecticides used for mosquito control rather than unmodified wild-type mosquitoes of the same genetic background.

The second workshop focused on efficacy and safety parameters that could be measured under containment in the laboratory, insectary, or indoor cage facility, and that would provide useful criteria for decision-making on candidates to move into field testing. Measures of gene drive construct functionality will be dependent upon whether the gene drive is intended for population replacement or population suppression. However, there was agreement that certain criteria would be important irrespective of the gene drive product.

Parameters such as mosquito numbers, mosquito longevity, vector competence, and human biting rate inform the calculation of vectorial capacity. Other parameters, such as homing rate, mating competitiveness, and reproductive success, will influence the performance of the gene drive system. Participants suggested that the most practical way to identify issues such as leaky transgene expression in nontarget tissues or off-target effects is by following fitness and other phenotypic changes, although in the case that problems are observed, developers may need to follow up with more extensive investigation to determine how to improve the construct. Development of resistance to the drive or effector mechanism would limit efficacy at the population level. Resistance could appear due to existing variations in the target sequence, nuclease-induced mutations, or random appearance of natural mutations. Participants proposed testing for functional resistance by conducting multiple crosses of heterozygous gene drive-modified and wild-type mosquitoes, and then examining the sequence at the gene drive target site in progeny that does not acquire the modification.

Participants recognized the need for an efficient and costeffective procedure for early identification of investigational products that should not be advanced to the field. For population suppression products, the recommended testing sequence was initial confirmation of biased inheritance in the F1 generation, followed by fitness measurements, and finally measurement of population decline in indoor cage studies. For population replacement products, the recommended experimental sequence was initial confirmation of biased inheritance, followed by testing of effector function in standard membrane feeding assays at high and low parasite concentrations, measurement of spread of the gene drive construct in cage studies, and finally measurement of other standard fitness parameters. Population replacement candidates should be tested against a variety of parasite lines as well as isolates from the proposed field site. Candidates that fail to meet designated criteria for a minimally acceptable product at any stage in this testing process would not be considered for advancement to field testing, but might return to the laboratory for further refinement.

TPP biosafety parameters for gene drive-modified mosquitoes are proposed in Table 3. Considerations for safety studies include identifying what data are required to meet regulatory requirements and gain public support, with the understanding that these needs may differ. These choices should be informed by case-by-case risk assessment. Based
Table 3. Proposed Biosafety Parameters for Target Product Profiles of Gene Drive-Modified Mosquitoes

1. Vector competence-predictor of safety for human and animal health

a. Plasmodium species

b. Selected other pathogens carried by Anopheles gambiae

2. Allergenicity or toxicity-predictor of safety for human and animal health

a. Codex Alimentarius guidelines

3. Behavior change-predictor of safety for human and animal health

a. Increased adult mosquito biting rate

b. Broader temperature tolerance

4. Insecticide susceptibility-predictor of safety for human and animal health

a. WHO guidelines

5. Ecosystem effect-predictor of safety for biodiversity a. Potential for unacceptable effect on nontarget species

b. Potential for unacceptable effect on ecosystem services

on experience with regulatory applications for genetically modified crops in Africa (National Biosafety Authority of Kenya; Convention on Biological Diversity 2000, Annex iii), anticipated requirements for contained use and field trials of gene drive-modified mosquitoes are likely to include information relating to the gene drive construct and phenotype, conditions of release and the potential receiving environment, interactions between the engineered gene drive and the environment, and environmental risk assessment. Evidence may come from experimental results, literature review, or modeling.

Participants agreed on the need for case-by-case risk assessment to identify relevant biosafety concerns. The most prominently identified hazards associated with gene drivemodified mosquitoes relate to biodiversity, human and animal health (Roberts et al. 2017, Teem et al. 2019). Health concerns can be directly addressed through laboratory studies to assess whether the gene drive construct increases vector competence for Plasmodium species and other key pathogens, causes changes that might result in increased toxicity or allergenicity, or increases host biting behavior. Participants proposed expansion of large-scale genetic screening of mosquito field populations as the most expedient method to collect information required to further parameterize models and answer risk-related questions about potential dispersal of gene drive-modified mosquitoes and gene flow among mosquito species.

Another often-raised concern is the potential for unplanned introduction of the construct into the genomes of other nontarget species. Consideration of possible nontarget effects should focus on which other organisms are locally valued (Convention on Biological Diversity 2019) and/or most likely to interact with gene drive-modified mosquitoes, as well as what harm could arise. Participants emphasized that spread of the construct to other organisms is not necessarily a harm in itself. Ecological impacts on nontarget species will require an understanding of how releases of gene drives might impact on parasites, competitors, and predators of mosquitoes. It was noted that there is no evidence that 
A. gambiae provide a critical food source for any other organism or are an important pollinator (Collins et al. 2019). The possibility that another undesirable organism could invade the ecological niche vacated as a result of population suppression strategies is a concern that should be addressed by risk assessment and monitored after initial release. However, it is important to recall that for this indication, the intent of population suppression strategies is to reduce numbers of vector mosquitoes to a level insufficient to maintain transmission of the malaria pathogen, not to eliminate the vector. Recent modeling suggests it is unlikely that population suppression strategies would completely eliminate the mosquito species under real-world conditions (North et al. 2019).

The workshops summarized in this study took an important step toward establishment of consensus criteria for PPCs and TPPs for gene drive-modified mosquitoes intended as a biocontrol tool for elimination of malaria in Sub-Saharan Africa. It was widely agreed that questions such as the minimal epidemiological efficacy goal should be raised with additional African malaria control experts, who will be the best judge of the needs for new tools to eliminate the disease there, and plans are underway to support further discussion.

The conclusions and recommendations arising from these discussions indicate that it will be possible to satisfy important requirements for the safety and efficacy evaluation of gene drive-modified mosquitoes that will form the basis for decision-making about moving from contained testing to first field release. It should be noted, however, that the methods suggested in this study are not exhaustive, and more direct or otherwise preferable alternative methods may become available as the research advances. It is hoped that the deliberations summarized in this study will be useful to developers and decision-makers as they consider the pathway for development of this potential new tool to achieve malaria elimination goals (WHO 2016b, African Union Commission 2018).

\section{Acknowledgments}

The authors thank the participants in the two 2019 workshops cited in this study for important discussions leading to this article.

\section{Author Disclosure Statement}

No conflicting financial interests exist.

\section{Funding Information}

The workshops were supported by grants OPP1161383 and OPP1128976 from the Bill \& Melinda Gates Foundation.

\section{References}

African Union Commission. African Union Malaria Progress Report, 2018. Available at https://endmalaria.org/sites/default/ files/African\%20Union\%20Malaria\%20Progress\%20Report\% 202019.pdf Accessed December 16, 2019.

Aguilar R, Dong Y, Warr E, Dimopoulos G. Anopheles infection responses; laboratory models versus field malaria transmission systems. Acta Trop 2005; 95:285-291.

Aldersley A, Pongsiri A, Bunmee K, Kijchalao U, et al. Too "sexy" for the field? Paired measures of laboratory and semifield performance highlight variability in the apparent mating fitness of Aedes aegypti transgenic strains. Parasit Vectors 2019; 12:357.

Amuzu H, Wilson MD, Boakye DA. Studies of Anopheles gambiae s.l (Diptera: Culicidae) exhibiting different vectorial capacities in lymphatic filariasis transmission in the Gomoa district, Ghana. Parasit Vectors 2010; 3:85.

Bayoh MN, Mathias DK, Odiere MR, Mutuku FM, et al. Anopheles gambiae: Historical population decline associated with regional distribution of insecticide-treated bed nets in western Nyanza Province, Kenya. Malar J 2010; 9:62.

Beaghton A, Hammond A, Nolan T, Crisanti A, et al. Requirements for driving antipathogen effector genes into populations of disease vectors by homing. Genetics 2017; 205:1587-1596.

Benedict MQ, Charlwood JD, Harrington LC, Lounibos LP, et al. Guidance for evaluating the safety of experimental releases of mosquitoes, emphasizing mark-release-recapture techniques. Vector Borne Zoonotic Dis 2018; 18:39-48.

Bradburd GS, Ralph PL, Coop GM. A spatial framework for understanding population structure and admixture. PLoS Genet 2016; 12:e1005703.

Brady OJ, Godfray HC, Tatem AJ, Gething PW, et al. Vectorial capacity and vector control: Reconsidering sensitivity to parameters for malaria elimination. Trans R Soc Trop Med Hyg 2016; 110:107-117.

Burrows J, Slater H, Macintyre F, Rees S, et al. A discovery and development roadmap for new endectocidal transmissionblocking agents in malaria. Malar J 2018; 17:462.

Burrows JN, Duparc S, Gutteridge WE, Hooft van Huijsduijnen $\mathrm{R}$, et al. New developments in anti-malarial target candidate and product profiles. Malar J 2017; 16:26.

Burt A, Trivers R. Genes in Conflict: The Biology of Selfish Genetic Elements. United States of America: Harvard University Press, 2006.

Carballar-Lejarazu R, James AA. Population modification of Anopheline species to control malaria transmission. Pathog Glob Health 2018; 111:1-12.

Champer J, Reeves R, Oh SY, Liu C, et al. Novel CRISPR/Cas9 gene drive constructs reveal insights into mechanisms of resistance allele formation and drive efficiency in genetically diverse populations. PLoS Genet 2017; 13:e1006796.

Collins CM, Bonds JAS, Quinlan MM, Mumford JD. Effects of the removal or reduction in density of the malaria mosquito, Anopheles gambiae s.1., on interacting predators and competitors in local ecosystems. Med Vet Entomol 2019; 33: $1-15$.

Convention on Biological Diversity. The Cartagena Protocol on Biosafety. 2000. Available at https://bch.cbd.int/protocol Accessed December 16, 2019.

Convention on Biological Diversity. National Biodiversity Strategies and Action Plans (NBSAPs). 2019. Available at www.cbd.int/nbsap Accessed December 11, 2019.

Coulibaly MB, Gabriel EE, Sinaba Y, Sylla D, et al. Optimizing direct membrane and direct skin feeding assays for Plasmodium falciparum transmission-blocking vaccine trials in Bancoumana, Mali. Am J Trop Med Hyg 2017; 97:719-725.

Dong Y, Das S, Cirimotich C, Souza-Neto JA, et al. Engineered anopheles immunity to Plasmodium infection. PLoS Pathog 2011; 7:e1002458.

Drugs for Neglected Diseases Initiative. Target Product Profiles. Available at www.dndi.org/diseases-projects/targetproduct-profiles Accessed December 11, 2019.

Dye C. Vectorial capacity: Must we measure all its components? Parasitol Today 1986; 2:203-209. 
Eckhoff PA, Wenger EA, Godfray HC, Burt A. Impact of mosquito gene drive on malaria elimination in a computational model with explicit spatial and temporal dynamics. Proc Natl Acad Sci U S A 2017; 114:E255-E264.

Erickson SM, Xi Z, Mayhew GF, Ramirez JL, et al. Mosquito infection responses to developing filarial worms. PLoS Negl Trop Dis 2009; 3:e529.

Facchinelli L, Valerio L, Ramsey JM, Gould F, et al. Field cage studies and progressive evaluation of genetically-engineered mosquitoes. PLoS Negl Trop Dis 2013; 7:e2001.

Fontaine MC, Pease JB, Steele A, Waterhouse RM, et al. Mosquito genomics. Extensive introgression in a malaria vector species complex revealed by phylogenomics. Science 2015; 347:1258524.

Food Allergy Research and Resource Program. Updated bioinformatics analysis for risks of allergenicity and toxicity of proteins encoded by the two genes introducted into genetically engineered mosquitoes (Aedes aegypti), strain OX513A for production of sterile males to reduce vector transmission of important human diseases. 2017. Available at www.rivm.nl/bibliotheek/rapporten/2017-0087_AI2 Accessed December 17, 2019.

Food and Agriculture Organization. Codex Alimentarius. Guidelines, Available at www.fao.org/fao-who-codexalimentarius/ codex-texts/guidelines/en Accessed December 11, 2019.

Foy BD, Alout H, Seaman JA, Rao S, et al. Efficacy and risks of harms of repeat ivermectin mass drug administration for control of malaria (RIMDAMAL): A cluster-randomized trial. Lancet 2019; 393:1517-1526.

Gantz VM, Jasinskiene N, Tatarenkova O, Fazekas A, et al. Highly efficient Cas9-mediated gene drive for population modification of the malaria vector mosquito Anopheles stephensi. Proc Natl Acad Sci U S A 2015; 112:E6736-E6743.

Gillies M, Smith A. The effect of a residual house-spraying campaign in East Africa on species balance in the Anopheles funestus group. The replacement of A. funestus Giles by A. rivulorum Leeson. Bull Entomol Res 1960; 51:243-252.

Godfray HCJ, North A, Burt A. How driving endonuclease genes can be used to combat pests and disease vectors. BMC Biol 2017; 15:81.

Griffin JT, Ferguson NM, Ghani AC. Estimates of the changing age-burden of Plasmodium falciparum malaria disease in subSaharan Africa. Nat Commun 2014; 5:3136.

Griffin JT, Hollingsworth TD, Okell LC, Churcher TS, et al. Reducing Plasmodium falciparum malaria transmission in Africa: A model-based evaluation of intervention strategies. PLoS Med 2010; 7. pii: e1000324.

Hammond A, Galizi R, Kyrou K, Simoni A, et al. A CRISPRCas9 gene drive system targeting female reproduction in the malaria mosquito vector Anopheles gambiae. Nat Biotechnol 2016; 34:78-83.

Hammond A, Kyrou K, Gribble M, Karlsson X, et al. Improved CRISPR-based suppression gene drives mitigate resistance and impose a large reproductive load on laboratory-contained mosquito populations. bioRxiv 2018:360339. DOI: https:// doi.org/10.1101/360339

Hammond AM, Galizi R. Gene drives to fight malaria: Current state and future directions. Pathog Glob Health 2017;111: 412-423.

Hammond AM, Kyrou K, Bruttini M, North A, et al. The creation and selection of mutations resistant to a gene drive over multiple generations in the malaria mosquito. PLoS Genet 2017; 13:e1007039.
International Union for Conservation of Nature and Natural Resources. The IUCN Red List of Threatened Species. 2019. Available at www.iucnredlist.org Accessed December 11, 2019.

Irvine MA, Reimer LJ, Njenga SM, Gunawardena S, et al. Modelling strategies to break transmission of lymphatic filariasis-Aggregation, adherence and vector competence greatly alter elimination. Parasit Vectors 2015; 8:547.

James S, Collins FH, Welkhoff PA, Emerson C, et al. Pathway to deployment of gene drive mosquitoes as a potential biocontrol tool for elimination of malaria in Sub-Saharan Africa: Recommendations of a Scientific Working Group. Am J Trop Med Hyg 2018; 98(6_Suppl):1-49.

James S, Takken W, Collins FH, Gottlieb M. Needs for monitoring mosquito transmission of malaria in a pre-elimination world. Am J Trop Med Hyg 2014; 90:6-10.

Jasper M, Schmidt TL, Ahmad NW, Sinkins SP, et al. A genomic approach to inferring kinship reveals limited intergenerational dispersal in the yellow fever mosquito. Mol Ecol Resour 2019; 19:1254-1264.

Kitau J, Oxborough RM, Tungu PK, Matowo J, et al. Species shifts in the Anopheles gambiae complex: Do LLINs successfully control Anopheles arabiensis? PLoS One 2012; 7: e31481.

Kleinstiver BP, Pattanayak V, Prew MS, Tsai SQ, et al. Highfidelity CRISPR-Cas9 nucleases with no detectable genomewide off-target effects. Nature 2016; 529:490-495.

Kyrou K, Hammond AM, Galizi R, Kranjc N, et al. A CRISPRCas9 gene drive targeting doublesex causes complete population suppression in caged Anopheles gambiae mosquitoes. Nat Biotechnol 2018; 36:1062-1066.

Lee Y, Marsden CD, Norris LC, Collier TC, et al. Spatiotemporal dynamics of gene flow and hybrid fitness between the $\mathrm{M}$ and $\mathrm{S}$ forms of the malaria mosquito, Anopheles gambiae. Proc Natl Acad Sci U S A 2013; 110:1985419859.

Marshall JM, Buchman A, Sanchez CHM, Akbari OS. Overcoming evolved resistance to population-suppressing homingbased gene drives. Sci Rep 2017; 7:3776.

McGreevy PB, Bryan JH, Oothuman P, Kolstrup N. The lethal effects of the cibarial and pharyngeal armatures of mosquitoes on microfilariae. Trans R Soc Trop Med Hyg 1978; 72 : 361-368.

Miura K, Deng B, Tullo G, Diouf A, et al. Qualification of standard membrane-feeding assay with Plasmodium falciparum malaria and potential improvements for future assays. PLoS One 2013; 8:e57909.

Moreira LA, Wang J, Collins FH, Jacobs-Lorena M. Fitness of anopheline mosquitoes expressing transgenes that inhibit Plasmodium development. Genetics 2004; 166:1337-1341.

National Academies of Science Engineering and Medicine. Gene Drives on the Horizon Advancing Science, Navigating Uncertainty, and Aligning Research with Public Values. 2016. Available at http://nas-sites.org/gene-drives Accessed December 16, 2019.

National Biosafety Authority of Kenya. Application Forms. Available at https://biosafetykenya.go.ke/index.php?option= com_content\&view=article\&id=29\&Itemid=131 Accessed December 11, 2019.

Ng'habi KR, Lee Y, Knols BG, Mwasheshi D, et al. Colonization of malaria vectors under semi-field conditions as a strategy for maintaining genetic and phenotypic similarity with wild populations. Malar J 2015; 14:10. 
North AR, Burt, Godfray HCJ. Modelling the potential of genetic control of malaria mosquitoes at national scale. BMC Biol 2019; 17:26.

Penny MA, Verity R, Bever CA, Sauboin C, et al. Public health impact and cost-effectiveness of the RTS,S/AS01 malaria vaccine: A systematic comparison of predictions from four mathematical models. Lancet 2016; 387:367-375.

Pike A, Dimopoulos G. Genetic modification of Anopheles stephensi for resistance to multiple Plasmodium falciparum strains does not influence susceptibility to o'nyong'nyong virus or insecticides, or Wolbachia-mediated resistance to the malaria parasite. PLoS One 2018; 13:e0195720.

Pioneer Hi-Bred International, Inc. Seed Production Technology (SPT) Process DP-32138-1, final risk assessment. 2011. Available at https://www.aphis.usda.gov/brs/aphisdocs/08_33801p_ fea.pdf Accessed February 25, 2020.

Raybould A. Ecological versus ecotoxicological methods for assessing the environmental risks of transgenic crops. Plant Sci 2007; 173:589-602.

Richards HA, Han CT, Hopkins RG, Failla ML, et al. Safety assessment of recombinant green fluorescent protein orally administered to weaned rats. J Nutr 2003; 133:1909-1912.

Roberts A, Andrade PP, Okumu F, Quemada H, et al. Results from the workshop "problem formulation for the use of gene drive in mosquitoes". Am J Trop Med Hyg 2017; 96:530 533.

Smith DL, McKenzie FE. Statics and dynamics of malaria infection in Anopheles mosquitoes. Malar J 2004; 3:13.

Smith DL, McKenzie FE, Snow RW, Hay SI. Revisiting the basic reproductive number for malaria and its implications for malaria control. PLoS Biol 2007; 5:e42.

Teem JL, Ambali A, Glover B, Ouedraogo J, et al. Problem formulation for gene drive mosquitoes designed to reduce malaria transmission in Africa: Results from four regional consultations 2016-2018. Malar J 2019; 18:347.

Unckless RL, Clark AG, Messer PW. Evolution of resistance against CRISPR/Cas9 gene drive. Genetics 2017; 205:827841.

US Department of Health Education and Welfare. Protection of Human subjects; Notice of Report for Public Comment. 1979. Available at https://www.hhs.gov/ohrp/regulations-and-policy/ belmont-report/read-the-belmont-report/index.html Accessed December 16, 2019.

Vanlandingham DL, Hong C, Klingler K, Tsetsarkin K, et al. Differential infectivities of o'nyong-nyong and chikungunya virus isolates in Anopheles gambiae and Aedes aegypti mosquitoes. Am J Trop Med Hyg 2005; 72:616-621.

Vontas J, Moore S, Kleinschmidt I, Ranson H, et al. Framework for rapid assessment and adoption of new vector control tools. Trends Parasitol 2014; 30:191-204.

Woolhouse ME, Dye C, Etard JF, Smith T, et al. Heterogeneities in the transmission of infectious agents: Implications for the design of control programs. Proc Natl Acad Sci U S A 1997; 94:338-342.

World Health Organization. Guidance framework for testing of genetically modified mosquitoes. 2014a. Available at http:// apps.who.int/iris/bitstream/10665/127889/1/9789241507486_ eng.pdf?ua=1 Accessed December 16, 2019.

World Health Organization. WHO preferred product characteristics (PPC) for malaria vaccines. 2014b. Available at
https://apps.who.int/iris/bitstream/handle/10665/149822/WHO_ IVB_14.09_eng.pdf; Accessed December 16, 2019.

World Health Organization. Guidelines for the Treatment of Malaria. 3rd edition, Annex 2, Malaria transmission and antimalarial medicines. 2015. Available at www.ncbi.nlm.nih .gov/books/NBK294437/ Accessed December 13, 2019.

World Health Organization. Test Procedures for Insecticide Resistance Monitoring in Malaria Vector Mosquitoes, 2nd ed. 2016a. Available at https://apps.who.int/iris/bitstream/handle/ 10665/250677/9789241511575-eng.pdf; Accessed December 16, 2019.

World Health Organization. Eliminating malaria. 2016b. Available at: https://apps.who.int/iris/bitstream/handle/10665/ 205565/WHO_HTM_GMP_2016.3_eng.pdf; Accessed December 16, 2019.

World Health Organization. WHO malaria terminology. 2016c. Available at https://apps.who.int/iris/bitstream/handle/10665/ 208815/WHO_HTM_GMP_2016.6_eng.pdf?sequence=1 Accessed January 18, 2020.

World Health Organization. Design of epidemiological trials for vector control products. 2017a. Available at: https://apps.who .int/iris/bitstream/handle/10665/255854/WHO-HTM-NTDVEM-2017.04-eng.pdf?sequence=1 Accessed December 16, 2019.

World Health Organization. How to design vector control efficacy trials. 2017b. Available at https://apps.who.int/iris/ bitstream/handle/10665/259688/WHO-HTM-NTD-VEM-2017 .03-eng.pdf?sequence $=1$ Accessed January 18, 2020.

World Health Organization. Methodology for prioritizing severe emerging diseases for research and development. 2017c. Available at www.who.int/blueprint/priority-diseases/RDBlue print-PrioritizationTool.pdf Accessed December 16, 2019.

World Health Organization. Global vector control response 20172030. 2017d. Available at https://apps.who.int/iris/bitstream/ handle/10665/259205/9789241512978-eng.pdf?sequence $=1$ Accessed December 16, 2019.

World Health Organization. A framework for malaria elimination. 2017e. Available at https://apps.who.int/iris/bitstream/handle/ 10665/254761/9789241511988-eng.pdf; Accessed January 18, 2020.

World Health Organization. Malaria [elimination] Certification process. 2018a. Available at www.who.int/malaria/areas/ elimination/certification/en Accessed December 6, 2019.

World Health Organization. Malaria Surveillance, Monitoring \& Evaluation: A reference manual. 2018b. Available at: https:// apps.who.int/iris/bitstream/handle/10665/272284/97892415655 78-eng.pdf?ua=1 Accessed December 16, 2019.

Yang G, Kim D, Pham A, Paul CJ. A meta-regression analysis of the effectiveness of mosquito nets for malaria control: The value of long-lasting insecticide nets. Int $\mathrm{J}$ Environ Res Public Health 2018; 15. DOI: 10.3390/ijerph15030546

Address correspondence to: Stephanie L. James Foundation for the National Institutes of Health North Bethesda, MD 20852

E-mail: sjames@fnih.org 